\title{
Fuzzy Minimal Solution of Dual Fully Fuzzy Matrix Equations
}

\author{
Dequan Shang ${ }^{1}$ and Xiaobin Guo ${ }^{2, *}$ \\ ${ }^{1}$ Science Courses Teaching Department, Gansu Traditional Chinese Medicine University, Lanzhou 730000, China \\ ${ }^{2}$ College of Mathematics and Statistics, Northwest Normal University, Lanzhou 730070, China \\ *Corresponding author
}

\begin{abstract}
The paper deals with the dual fully fuzzy matrix

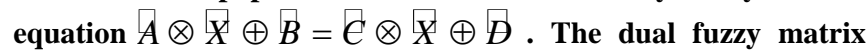
equation is converted to a crisp system of matrix equations according to arithmetic operations of fuzzy numbers. The fuzzy approximate solution of fuzzy matrix equation is obtained by solving the model which is made of three linear matrix equations. The existence condition of the nonnegative fuzzy solution is also discussed. A example is given to illustrate the proposed method.
\end{abstract}

Keywords-fuzzy numbers; matrix analysis; dual fuzzy matrix equations; fuzzy approximate solutions

\section{INTRODUCTION}

System of simultaneous linear matrix equations is an essential mathematical tool in science and technology. In practice some or all parameters may be represented by fuzzy numbers rather than crisp ones. Therefore it is necessary to develop mathematical theory and numerical schemes to handle fuzzy matrix systems. The concept of fuzzy numbers and arithmetic operations with these numbers were first introduced and investigated by Zadeh [1], Dubois et al.[2] and Nahmias [3].

Since Friedman et al. [4] proposed a general model for solving an fuzzy linear systems $A x=b$ by an embedding approach in 1998, lots of works have been done about some advanced fuzzy linear systems such as dual fuzzy linear systems (DFLS), general fuzzy linear systems (GFLS), fully fuzzy linear systems (FFLS)and general dual fuzzy linear systems (GDFLS) see [5-8]. And some new theories and methods for fuzzy linear systems still appeared recently [9-11].

However, for a fuzzy linear matrix equation which always has a wide use in control theory and control engineering, few work has been done in the past decades. In 2009 , Allahviranloo et al. [12] discussed the fuzzy linear matrix equations(FLME) of the form $A X B=€$ where $A$ and $B$ are $m \times m$ and $n \times n$ real matrices respectively, $\ell$ is a given $m \times n$ fuzzy numbers matrix. In 2011 , Gong and Guo [13] investigated a class of fuzzy matrix equations $\tilde{A x}=B$ and studied its fuzzy least squares solutions by using generalized inverses of the matrix. Later, Guo and Shang [14-15] proposed a computing method of fuzzy symmetric solutions to fuzzy matrix equations $A X=B$ and discussed the fuzzy Sylvester matrix equations with $L R$ fuzzy numbers. Recently, Guo and Gong
[16] investigated the dual fuzzy matrix equations form $A X+B=C X+D$ based on LR fuzzy numbers.

\section{II.PRELIMINARIES}

\section{A. LR Fuzzy Numbers}

Let $E^{1}$ be the set of all fuzzy numbers on $R$.

Definition 2.1. A fuzzy number $M$ is said to be a $L R$ fuzzy number if

$$
u_{M}(x)=\left\{\begin{array}{l}
L\left(\frac{m-x}{\alpha}\right), x \leq m, \alpha>0, \\
R\left(\frac{x-m}{\beta}\right), x \geq m, \beta>0,
\end{array}\right.
$$

where $m$ is the mean value of $M$, and $\alpha$ and $\beta$ are left and right spreads, respectively. The function $L(\cdot)$; which is called left shape function satisfying:

(1) $L(x)=L(-x)$,

(2) $L(0)=1$ and $L(1)=0$,

(3) $L(x)$ is nonincreasing on $[0, \infty)$.

The definition of a right shape function $R(\cdot)$ is usually similar to that of $L(\cdot)$.A $L R$ fuzzy number $M$ is symbolically shown as $M=(m, \alpha, \beta)_{L R}$.

Clearly, $M=(m, \alpha, \beta)_{L R}$ is positive(negative) if and only if $m-\alpha>0(m+\beta<0)$.

Definition 2.2. For arbitrary $L R$ fuzzy numbers $M=(m, \alpha, \beta)_{L R}$ and $\quad \mathrm{N}=(n, \gamma, \delta)_{L R}$, we have

(1) Addition 
$M \oplus N=(m, \alpha, \beta)_{L R} \oplus(n, \gamma, \delta)_{L R}=(m+n, \alpha+\gamma, \beta+\delta)_{L R}$

(2) Multiplication

If $M>0$ and $N>0$; then

$M \otimes N=(m, \alpha, \beta)_{L R} \otimes(n, \gamma, \delta)_{L R}=(m n, m \gamma+n \alpha, m \delta+n \beta)_{L R}$

\section{B. The Dual Fully Fuzzy Matrix Equations}

Definition 2.3. A matrix $A=\left(\tilde{a}_{i j}\right), 1 \leq i \leq m, 1 \leq j \leq n$ is called a $L R$ fuzzy matrix, if each element of $A$ is a $L R$ fuzzy number.

A $L R$ fuzzy matrix $A$ is said to be positive(negative) and denoted by $A>0(A<0)$ if each element $a_{i j}, 1 \leq i \leq m, 1 \leq j \leq n$ of $\AA$ be positive (negative). We can represent $m \times n$ fuzzy matrix $\AA=\left(\tilde{a}_{i j}\right)$, that $a_{i j}=\left(a_{i j}, \alpha_{i j}, \beta_{i j}\right)_{L R}$ with new notation $A=(A, M, N)$, where $A=\left(a_{i j}\right), M=\left(\alpha_{i j}\right)$ and $N=\left(\beta_{i j}\right)$ are three $m \times n$ crisp matrices.

Definition 2.4. The linear system

$$
\begin{aligned}
\left(\begin{array}{llll}
a_{11} & a_{12} & \ldots & a_{1 n} \\
a_{21} & a_{22} & \ldots & a_{2 n} \\
\ldots & \ldots & \ldots & \ldots \\
a_{m 1} & a_{m 2} & \ldots & a_{m n}
\end{array}\right)\left(\begin{array}{llll}
x_{11} & x_{12} & \ldots & x_{1 p} \\
x_{21} & \chi_{22} & \ldots & x_{2 p} \\
\ldots & \ldots & \ldots & \ldots \\
x_{n 1} & x_{n 2} & \ldots & x_{n p}
\end{array}\right) \\
+\left(\begin{array}{cccc}
b_{11} & b_{12} & \ldots & b_{1 p} \\
b_{21} & b_{22} & \ldots & b_{2 p} \\
\ldots & \ldots & \ldots & \ldots \\
b_{m 1} & b_{m 2} & \ldots & b_{m p}
\end{array}\right)
\end{aligned}
$$

$$
\begin{aligned}
& =\left(\begin{array}{cccc}
e_{11} & e_{12} & \ldots & e_{1 n} \\
e_{21} & e_{22} & \ldots & e_{2 n} \\
\ldots & \ldots & \ldots & \ldots \\
e_{m 1} & c_{m 2} & \ldots & c_{m n}
\end{array}\right)\left(\begin{array}{llll}
x_{11} & x_{12} & \ldots & x_{1 p} \\
x_{21} & x_{22} & \ldots & x_{2 p} \\
\ldots & \ldots & \ldots & \ldots \\
x_{n 1} & x_{n 2} & \ldots & x_{n p}
\end{array}\right), \\
& +\left(\begin{array}{cccc}
d_{11} & d_{12} & \ldots & d_{1 p} \\
d_{21} & d_{22} & \ldots & d_{2 p} \\
\ldots & \ldots & \ldots & \ldots \\
d_{m 1} & d_{m 2} & \ldots & d_{m p}
\end{array}\right)
\end{aligned}
$$

where

$$
a_{i j}, c_{i j}, 1 \leq i \leq m, 1 \leq j \leq n
$$

and

$b_{i j}, d_{i j}, 1 \leq i \leq m, 1 \leq j \leq p$ are $L R$ fuzzy numbers, is called a $L R$ dual fully fuzzy matrix equations(DFFMEs).

Using matrix notation, we have

$$
A \otimes X \oplus B=€ \otimes X \oplus D .
$$

A $L R$ fuzzy numbers matrix $X=\left(X_{i j}\right)=\left(x_{i j}, y_{i j}, z_{i j}\right)_{L R}, 1 \leq i \leq n, 1 \leq j \leq p$ is said to the $L R$ solution of dual fuzzy matrix equation (2.1) if $X$ satisfies Eq. (2.2) , i.e.,

$$
\begin{aligned}
& A \otimes X_{j} \oplus B_{j}=E \otimes X_{j} \oplus D_{j}, j=1, \ldots, p ; \\
& X_{j}=\left(X_{1 j}, x_{2 j}, \ldots, x_{n j}\right)^{T}, B_{j}=\left(b_{1 j}, b_{2 j}, \ldots, b_{m j}\right)^{T}, \\
& D_{j}=\left(d_{1 j}, d_{2 j}, \ldots, d_{m j}\right)^{T}, j=1, \ldots, p
\end{aligned}
$$

are jth column of $L R$ fuzzy matrices $X, B$ and $D$ respectively.

Up to rest of this paper we want to find the positive solution $X=(X, Y, Z) \geq 0$ of nonnegative (2.2) , where $A=(A, M, N) \geq 0, B=(B, E, F) \geq 0, \mathcal{E}=(C, G, H) \geq 0$ and $D=(D, P, Q) \geq 0$.

\section{Solving DuAl Fully FuZzy MATRIX EQUATION}

Theorem 3.1. The dual fully fuzzy matrix system (2.2) can be extended into the following model system which is made of three linear matrix equations 


$$
\left\{\begin{array}{c}
A X+B=C X+D, \\
A Y+M X+E=C Y+G X+P, \\
A Z+N X+F=C Z+H X+Q .
\end{array}\right.
$$

\section{Proof.}

We

denote

$A=(A, M, N), B=(B, E, F), \mathcal{C}=(C, G, H), D=(D, P, Q)$

and assume $X=(X, Y, Z) \geq 0$, then

\section{$A \otimes X \oplus B=E \otimes X \oplus D$}

i.e.,

$(A, M, N) \otimes(X, Y, Z) \oplus(B, E, F)=(C, G, H) \otimes(X, Y, Z) \oplus(D, P, Q)$. According to multiplication of nonnegative LR fuzzy numbers of Definition 2.3 , we have

$(A X, A Y+M X, A Z+N X)+(B, F)=(X, C Y+G X, C Z+H X)+(D, P, Q)$. Thus we obtain a model for solving DFFMEs (2.2) as follows:

$$
\left\{\begin{array}{c}
A X+B=C X+D \\
A Y+M X+E=C Y+G X+P, \\
A Z+N X+F=C Z+H X+Q .
\end{array}\right.
$$

In order to solve the dual fuzzy system (2.2), we need to consider the crisp system of linear matrix equation (3.1). For example, when $A-C$ is a nonsingular crisp matrix, we obtain its solution as follows:

$$
\left\{\begin{array}{c}
X=(A-C)^{-1}(D-B) \\
Y=(A-C)^{-1}\left((P-E)-(G-M)(A-C)^{-1}(B-D)\right),(3.2) \\
Z=(A-C)^{-1}\left((Q-F)-(H-N)(A-C)^{-1}(B-D)\right) .
\end{array}\right.
$$

Theorem 3.2([17]). Let $A$ belong to $R^{m \times n}$ and $B$ belong to $R^{m \times p}$. Then the minimal solution $X^{*}$ of the matrix equation $A X=B$ is expressed by

$$
X^{*}=A^{\dagger} B
$$

When $A-C$ is a singular matrix, by the Theorem 3.2., we solve model (3.1) and obtain its minimal solution as follows:

$$
\left\{\begin{array}{c}
X=(A-C)^{\dagger}(D-B), \\
Y=(A-C)^{\dagger}\left((P-E)-(G-M)(A-C)^{\dagger}(B-D)\right),(3.3) \\
Z=(A-C)^{\dagger}\left((Q-F)-(H-N)(A-C)^{\dagger}(B-D)\right) .
\end{array}\right.
$$

Definition 3.2. Let $X=(X, Y, Z)$. If $X=(X, Y, Z)$

is the minimal solution of Eqs.(3.1) such that $X \geq 0, Y \geq 0, Z \geq 0$, then we say $X=(X, Y, Z)$ is a LR fuzzy minimal solution of Eqs.(2.2). If $X=(X, Y, Z)$ also satisfies $X-Y \geq 0$, we say it is a nonnegative $L R$ fuzzy minimal solution of Eqs.(2.2).

Theorem 3.3. Let $A=(A, M, N), B=(B, E, F)$ and $E=(C, G, H)$ be three nonnegative $L R$ fuzzy matrices, respectively, and $(A-C)^{\dagger}$ be nonnegative matrix. Let $\quad D-B \geq 0, P-E \geq(G-M)(A-C)^{-1}(D-B)$ and $Q-F \geq(H-N)(A-C)^{-1}(D-B)$. Then the dual fuzzy systems $A \otimes X \oplus B=C \otimes X \oplus D$ has a $L R$ fuzzy minimal solution. If the condition $(D-B)\left(I+(G-M)(A-C)^{-1}\right) \geq(P-E)$ holds, then the dual fuzzy systems (2.1) has a nonnegative LR fuzzy minimal solution.

Proof. Since $(A-C)^{\dagger}$ and $D-B$ are nonnegative matrices. Thus the fact $X=(A-C)^{\dagger}(D-B) \geq 0$ is apparent.

On the other hand, because $P-E \geq(G-M)(A-C)^{\dagger}(D-B)$

and

$$
Q-F \geq(H-N)(A-C)^{\dagger}(D-B),
$$

so with $Y=(A-C)^{\dagger}\left((P-E)-(G-M)(A-C)^{\dagger}(B-D)\right)$ and

$$
Z=(A-C)^{\dagger}\left((Q-F)-(H-N)(A-C)^{\dagger}(D-B)\right),
$$

we have $Y \geq 0$ and $Z \geq 0$. Thus $X=(X, Y, Z)$ is a $L R$ fuzzy solution of the dual fuzzy systems $A \otimes X \oplus B=E \otimes X \oplus D$. Since 


$$
X-Y=(A-C)^{\dagger}\left((D-B)-(P-E)+(G-M)(A-C)^{\dagger}(D-B)\right),
$$

the nonnegative property of the solution $X$ to dual fuzzy systems (2.1) can be obtained from the condition $\left(I+(G-M)(A-C)^{\dagger}(D-B)\right) \geq(P-E)$.

\section{NUMERICAL EXAMPLE}

Example 4.1. Consider the dual fully fuzzy matrix system

$$
\begin{aligned}
& \left(\begin{array}{lll}
(2,1,1)_{L R} & (3,1,2)_{L R} & (1,0,0)_{L R} \\
(3,1,1)_{L R} & (2,0,1)_{L R} & (2,1,1)_{L R}
\end{array}\right)\left(\begin{array}{ll}
x_{11} & x_{12} \\
x_{21} & \chi_{22} \\
x_{31} & \chi_{32}
\end{array}\right) \\
& +\left(\begin{array}{cc}
(2,2,1)_{L R} & (1,1,2)_{L R} \\
(2,1,1)_{L R} & (3,1,1)_{L R}
\end{array}\right) \\
& =\left(\begin{array}{lll}
(2,1,1)_{L R} & (1,0,0)_{L R} & (1,1,1)_{L R} \\
(1,0,1)_{L R} & (2,1,1)_{L R} & (3,0,1)_{L R}
\end{array}\right)\left(\begin{array}{ll}
x_{11} & x_{12} \\
x_{21} & x_{22} \\
x_{31} & \chi_{32}
\end{array}\right) \\
& +\left(\begin{array}{cc}
(4,2,2)_{L R} & (3,2,2)_{L R} \\
(5,4,3)_{L R} & (3,2,0)_{L R}
\end{array}\right) .
\end{aligned}
$$

By Theorem 3.1., the model to above fuzzy linear matrix system is made of following three crisp systems of linear matrix equations

$$
\left(\begin{array}{lll}
2 & 3 & 1 \\
3 & 2 & 2
\end{array}\right)\left(\begin{array}{ll}
x_{11} & x_{12} \\
x_{21} & x_{22} \\
x_{31} & x_{32}
\end{array}\right)+\left(\begin{array}{ll}
2 & 1 \\
2 & 3
\end{array}\right)=\left(\begin{array}{lll}
2 & 1 & 1 \\
1 & 2 & 3
\end{array}\right)\left(\begin{array}{ll}
x_{11} & x_{12} \\
x_{21} & x_{22} \\
x_{31} & x_{32}
\end{array}\right)+\left(\begin{array}{ll}
4 & 3 \\
5 & 3
\end{array}\right),
$$$$
\left(\begin{array}{lll}
2 & 3 & 1 \\
3 & 2 & 2
\end{array}\right)\left(\begin{array}{ll}
y_{11} & y_{12} \\
y_{21} & y_{22} \\
y_{31} & y_{32}
\end{array}\right)+\left(\begin{array}{ll}
2 & 1 \\
2 & 3
\end{array}\right)=\left(\begin{array}{lll}
2 & 1 & 1 \\
1 & 2 & 3
\end{array}\right)\left(\begin{array}{ll}
x_{11} & x_{12} \\
x_{21} & x_{22} \\
x_{31} & x_{32}
\end{array}\right)+\left(\begin{array}{ll}
2 & 1 \\
1 & 1
\end{array}\right)
$$$$
=\left(\begin{array}{lll}
2 & 1 & 1 \\
1 & 2 & 3
\end{array}\right)\left(\begin{array}{ll}
y_{11} & y_{12} \\
y_{21} & y_{22} \\
y_{31} & y_{32}
\end{array}\right)+\left(\begin{array}{lll}
1 & 0 & 1 \\
0 & 1 & 0
\end{array}\right)\left(\begin{array}{ll}
x_{11} & x_{12} \\
x_{21} & x_{22} \\
x_{31} & x_{32}
\end{array}\right)+\left(\begin{array}{ll}
2 & 2 \\
4 & 2
\end{array}\right)
$$

and

$$
\left(\begin{array}{lll}
2 & 3 & 1 \\
3 & 2 & 2
\end{array}\right)\left(\begin{array}{ll}
z_{11} & z_{12} \\
z_{21} & z_{22} \\
z_{31} & z_{32}
\end{array}\right)+\left(\begin{array}{lll}
1 & 2 & 0 \\
1 & 1 & 1
\end{array}\right)\left(\begin{array}{ll}
x_{11} & x_{12} \\
x_{21} & x_{22} \\
x_{31} & x_{32}
\end{array}\right)+\left(\begin{array}{ll}
1 & 2 \\
1 & 1
\end{array}\right)
$$

$$
\begin{aligned}
& =\left(\begin{array}{lll}
2 & 1 & 1 \\
1 & 2 & 3
\end{array}\right)\left(\begin{array}{ll}
z_{11} & z_{12} \\
z_{21} & z_{22} \\
z_{31} & z_{32}
\end{array}\right)+\left(\begin{array}{lll}
1 & 0 & 1 \\
1 & 1 & 1
\end{array}\right)\left(\begin{array}{ll}
x_{11} & x_{12} \\
x_{21} & x_{22} \\
x_{31} & x_{32}
\end{array}\right) \\
& +\left(\begin{array}{ll}
4 & 2 \\
3 & 0
\end{array}\right) .
\end{aligned}
$$

According to formula (3.3), the minimal solutions of above three systems of linear matrix equations are as follows:

$$
\begin{aligned}
X & =(A-C)^{\dagger}(D-B) \\
& =\left(\left(\begin{array}{lll}
2 & 3 & 1 \\
3 & 2 & 2
\end{array}\right)-\left(\begin{array}{lll}
1 & 0 & 1 \\
1 & 1 & 1
\end{array}\right)\right)^{\dagger}\left(\left(\begin{array}{ll}
4 & 3 \\
5 & 3
\end{array}\right)-\left(\begin{array}{ll}
2 & 1 \\
2 & 3
\end{array}\right)\right) \\
& =\left(\begin{array}{ll}
2.0571 & 1,2286 \\
1.3143 & 0.8429 \\
1.1327 & 2.2857
\end{array}\right)
\end{aligned}
$$

$$
\begin{aligned}
Y & =(A-C)^{\dagger}((P-E)-(G-M) X) \\
& =\left(\left(\begin{array}{lll}
2 & 3 & 1 \\
3 & 2 & 2
\end{array}\right)\left(\begin{array}{lll}
1 & 0 & 1 \\
1 & 1 & 1
\end{array}\right)\right)\left(\left(\left(\begin{array}{ll}
2 & 2 \\
4 & 2
\end{array}\right)\left(\begin{array}{ll}
2 & 1 \\
1 & 1
\end{array}\right)\right)\left(\left(\begin{array}{lll}
1 & 0 & 1 \\
0 & 1 & 0
\end{array}\right)\left(\begin{array}{lll}
1 & 1 & 0 \\
1 & 0 & 1
\end{array}\right)\right) X_{j}\right. \\
& =\left(\begin{array}{ll}
1.0333 & 0.1236 \\
01258 & 0.9896 \\
1.1250 & 1.3333
\end{array}\right)
\end{aligned}
$$

$$
\begin{aligned}
& Z=(A-C)^{\dagger}((Q-F)-(H-N) X) \\
& =\left(\left(\begin{array}{lll}
2 & 3 & 1 \\
3 & 2 & 2
\end{array}\right)-\left(\begin{array}{lll}
1 & 0 & 1 \\
1 & 1 & 1
\end{array}\right)\right)\left(\left(\left(\begin{array}{ll}
4 & 2 \\
3 & 0
\end{array}\right)-\left(\begin{array}{ll}
1 & 2 \\
1 & 1
\end{array}\right)\right)-\left(\left(\begin{array}{lll}
1 & 0 & 1 \\
1 & 1 & 1
\end{array}\right)-\left(\begin{array}{lll}
1 & 2 & 0 \\
1 & 1 & 1
\end{array}\right)\right) X\right) \\
& =\left(\begin{array}{ll}
1.0333 & 0.1236 \\
1.1258 & 0.0333 \\
0.1333 & 1.3333
\end{array}\right) \text {. }
\end{aligned}
$$


By Definition 3.2, we know the original fuzzy linear matrix equations has a nonnegative LR fuzzy solution

$$
X=\left(\begin{array}{ll}
x_{11} & x_{12} \\
x_{21} & x_{22} \\
x_{31} & x_{32}
\end{array}\right)=\left(\begin{array}{ll}
(20571,1,03331.0333)_{I R} & (1.296,0.12360 .1236)_{I R} \\
(1.3143,0.12580 .1258)_{I R} & (0.8429,0.98960 .0333)_{I R} \\
(1,1327,1.1250,0.1333)_{I R} & (22857,1.33331 .3333)_{I R}
\end{array}\right),
$$

Since $X \geq 0, Y \geq 0, Z \geq 0$ and $X-Y \geq 0$.

\section{CONCLUSION}

In this work, we proposed a simple model for solving the general dual fully fuzzy matrix equation. We converted the dual fuzzy matrix equation to a crisp system of linear matrix equations according to the arithmetic operations of $L R$ fuzzy numbers. The existence condition of nonnegative $L R$ fuzzy solution was also studied. Numerical examples showed that our method is effective to solve the dual fully fuzzy matrix equation.

\section{ACKNOWLEDGEMENTS}

This research was supported by the National Natural Science Foundation of China (no.61262022).

\section{REFERENCES}

[1] L.A. Zadeh, "The concept of a linguistic variable and its application to approximate reasoning”, Information Science 8 (1975) 199-249.

[2] D. Dubois, H. Prade, “Operations on fuzzy numbers”, Journal of Systems Science 9 (1978) 613-626.

[3] S. Nahmias, Fuzzy variables, "Fuzzy Sets and Systems”, 1(2)(1978) 97-111.

[4] M. Friedman, M. Ma, A. Kandel, "Fuzzy linear systems”, Fuzzy Sets and Systems 96 (1998) 201-209.

[5] M. Ma, M. Friedman, A. Kandel, "Duality in Fuzzy linear systems", Fuzzy Sets and Systems 109 (2000) 55-58.

[6] S. Abbasbandy, M. Otadi, M. Mosleh, "Minimal solution of general dual fuzzy linear systems”, Chaos, Solitions and Fractals 37 (2008) 1113-1124

[7] B. Zheng, K. Wang, "General fuzzy linear systems”, Applied Mathematics and Computation, 181 (2006) 1276-1286.

[8] M. Dehghan, B. Hashemi, M. Ghatee, "Solution of the full fuzzy linear systems using iterative techniques”, Chaos, Solitons and Fractals, 34 (2007) 316-336.

[9] T. Allahviranloo, M. Ghanbari, “A new approach to obtain algebraic solution of interval linear systems”, Soft Computing 16 (2013) 121-133.

[10] R. Ghanbari, N. Mahdavi-Amiri, "New solutions of LR fuzzy linear systems using ranking functions and ABS algorithms”, Applied Mathematical Modelling, 34 (2012) 3363-3375.

[11] R. Ghanbari, "Solutions of fuzzy LR algebraic linear systems using linear programs”, Applied Mathematical Modelling, 39 (2015) 5164-5173

[12] T. Allahviranloo, N. Mikaeilvand, M. Barkhordary, "Fuzzy linear matrix equations”, Fuzzy Optimization and Decision Making 8 (2009) 165-177.

[13] Z.T. Gong, X.B. Guo, “ Inconsistent fuzzy matrix equations and its fuzzy least squares solutions”, Applied Mathematical Modelling 35 (2011) 1456-1469.

[14] X.B. Guo, D.Q. Shang, "Fuzzy symmetric solutions of fuzzy matrix equations”, Advances in Fuzzy Systems, Volume 2012, Article ID 318069 9 pages.
[15] X.B. Guo, D.Q. Shang, “Approximate solutions of LR fuzzy Sylvester matrix equations”, Journal of Applied Mathematics, Volume 2013, Article ID 752760, 10 pages.

[16] Z.T. Gong, X.B. Guo, K. Liu, “Approximate solution of dual fuzzy matrix equations”, Information Sciences 266 (2014) 112-133.

[17] A. Berman, R.J. Plemmons, "Nonnegative matrices in the Mathematical Sciences”, Academic press, New York, 1979. 